\title{
Exploring mental rotation in schizotypy
}

\section{INTRODUCTION}

Findings from multiple fields support a link between schizotypal traits and schizophrenia (Ettinger et al., 2014). Furthermore, spatial working memory deficits have been found in people with high schizotypy (Hazlett et al., 2014).

Mental rotation (a spatial working memory skill) differences have been observed in people with schizophrenia (e.g., Chen et al., 2012), but results regarding schizotypy have been contradictory.

Thus, our goal was to explore the association between schizotypy and mental rotation by using a mental rotation task with varying difficulty levels, since schizotypy effects could be subtler than the ones found in schizophrenia.

Sex differences were also expected to be found in the task, a typical result in mental rotation paradigms (Boone, \& Hegarty, 2017).

\section{Participants}

149 students (81 female) from the

University of Almería.

\section{Materials}

- Schizotypal Personality

Questionnaire (SPQ; Raine, 1991). Cognitive-perceptual Interpersonal Disorganized

\section{METHOD}

a)

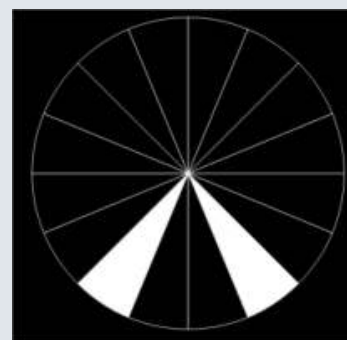

b)

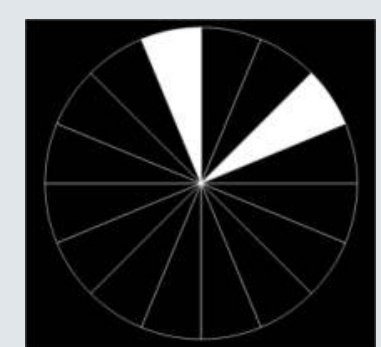

c)

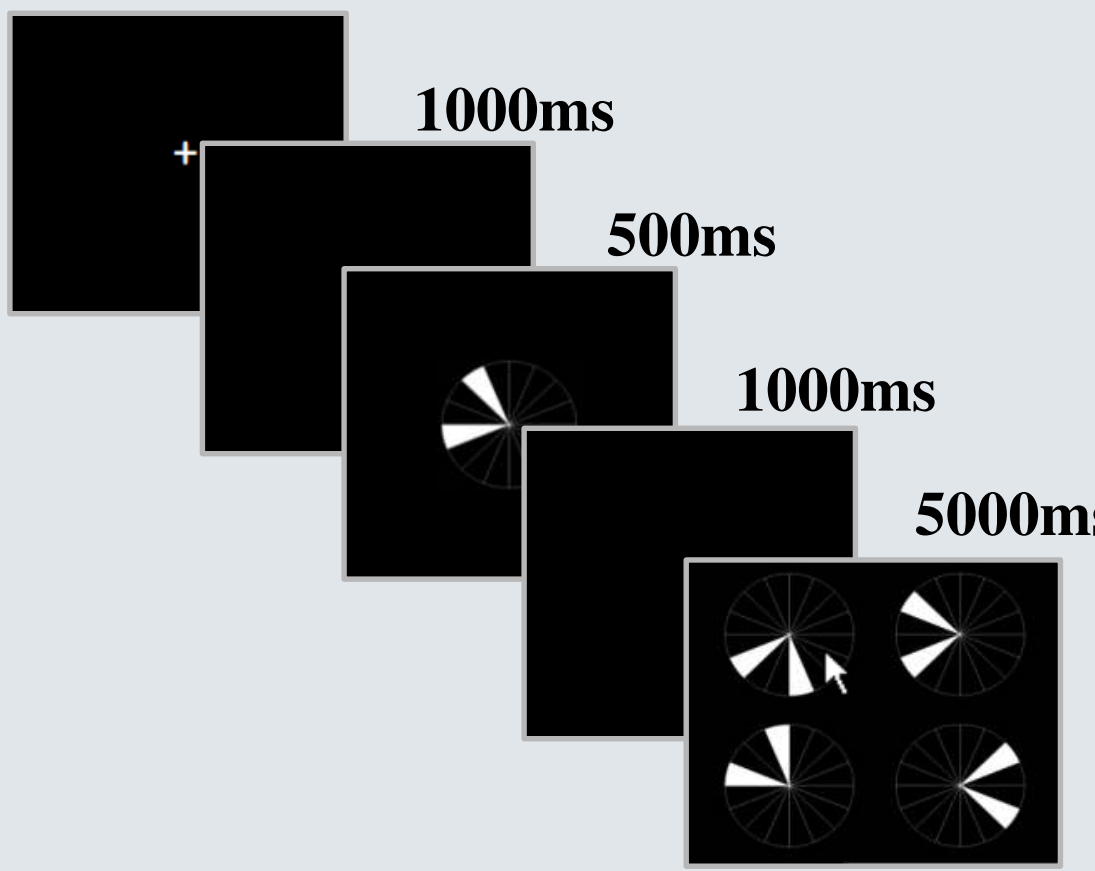

\section{RESULTS} Figure 2). found, where it was associated with better execution (see Figure 3 ).

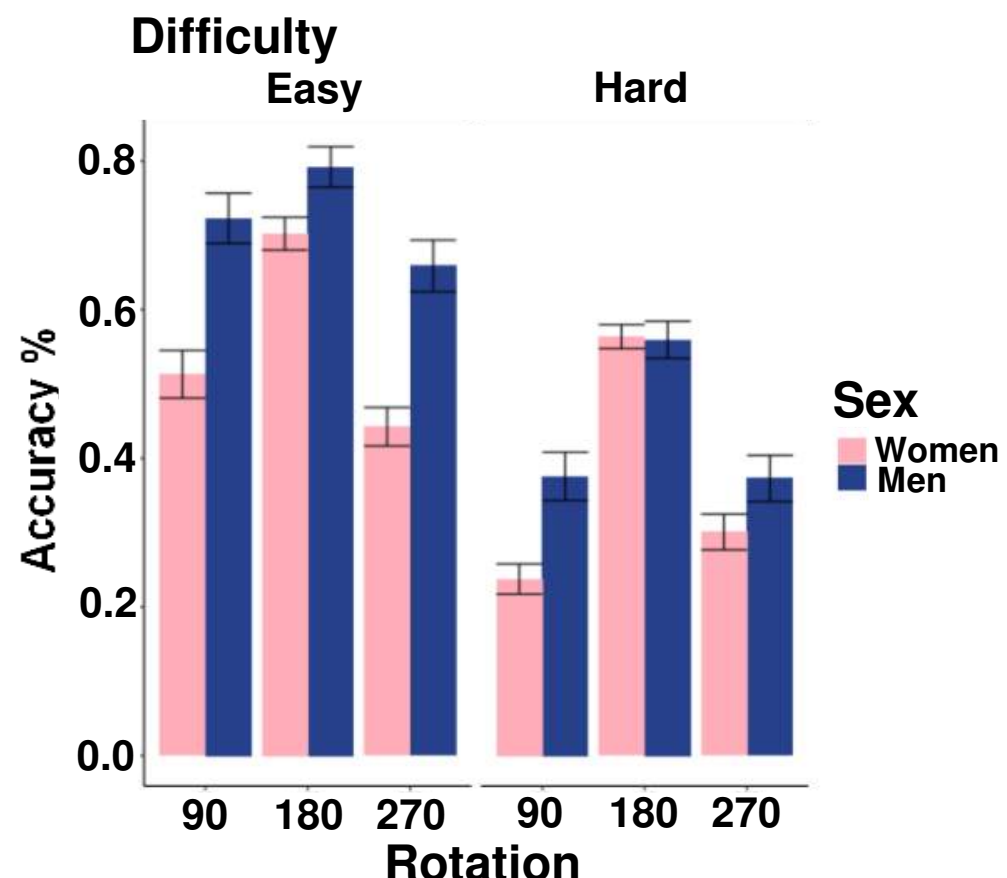

Figure 2. Mean accuracy comparison.

Participants had to identify the comparison stimulus that was a $90^{\circ}, 180^{\circ}$ or $270^{\circ}$ rotation from the sample stimulus.

\section{$10000 \mathrm{~ms}$ or} until response
- Black circles split into sixteen portions by eight axes; two of the portions coloured white.

- Easy and Hard sample stimuli.

- 90, 180 - and 270 ㅇtations.

Ref. Number. 1557

Welch-type statistics with trimmed means (10\%) and winsorized variances revealed a significant main effect of $\operatorname{Sex}\left(T_{W J}(1,89.2)=13.485, p=.001\right)$, and a significant sex $x$ difficulty interaction, with men performing better in both hard $\left(p=.015, \delta_{R}=0.248\right)$ and easy conditions $\left(p<.001, \delta_{R}=0.539\right)$. A significant sex $x$ degree interaction was also found: there were no significant sex differences only in 180 degree trials $(p=.14)$ (see

A Maximum Likelihood robust path análisis was carried out, using hard and easy dificulty accuracy as endogenous variables, and schizotypy factors as exogenous variables. An effect of the Disorganized factor on both easy $\left(R^{2}=.06\right)$ and hard $\left(R^{2}=.06\right)$ trial performance was

- 48 trials, no feedback.

Figure 1. Example of easy (a) and hard (b) sample stimuli, and trial diagram (c). References:

Boone, A. P., \& Hegarty, M. (2017). Sex differences in mental rotation tasks: Not just in the mental rotation process!. Journal of Experimental Psychology: Learning, Memory, and Cognition, 43(7), 1005-1019.
Chen, J., Yang, L., , Zhao, J., Li, L., Liu, G., Ma, W., . . . Tuo, R. (2012). Hemispheric dominance during the mental rotation task in patients with schizophrenia. Shanghai Archives of Psychiatry, 24(2), 76.

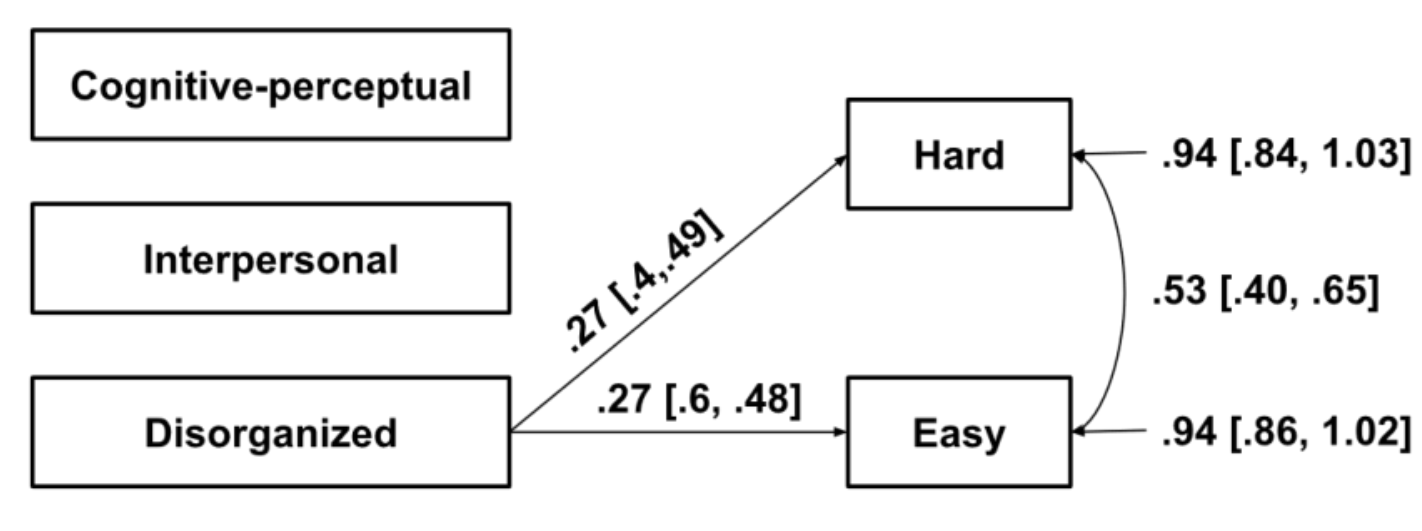

Figure 3. Path analysis diagram.

\section{DISCUSSION}

As expected, sex differences were found in the mental rotation task, except in the easiest of trials future studies should adress factors influencing them.

Disorganized factor of Schizotypy appeared to slightly improve mental rotation performance regardless of difficulty. More research is warranted to better characterize schizotypy effects on cognition. 